\title{
Adhesion between elastic solids with randomly rough surfaces: comparison of analytical theory with molecular dynamics simulations
}

\author{
N. Mulakaluri and B.N.J. Persson \\ Institut für FestköperForschung, Forschungszentrum Jülich, D-52425 Jülich, Germany
}

\begin{abstract}
The adhesive contact between elastic solids with randomly rough, self affine fractal surfaces is studied by molecular dynamics (MD) simulations. The interfacial binding energy obtained from the simulations of nominally flat and curved surfaces is compared with the predictions of the contact mechanics theory by Persson. Theoretical and simulation results agree rather well, and most of the differences observed can be attributed to finite size effects and to the long-range nature of the interaction between the atoms in the block and the substrate in the MD model, as compared to the analytical theory which is for an infinite system with interfacial contact interaction. For curved surfaces (JKR-type of problem) the effective interfacial energy exhibit a weak hysteresis which may be due to the influence of local irreversible detachment processes in the vicinity of the opening crack tip during pull-off.
\end{abstract}

Surface forces play an important role in modern technology which deals with micro/nano scale devices. Electromechanical devices on this length scale have undergone a rapid development within the last decade. The predominant effect of surface forces is due to the increase of the ratio between the number of atoms on the surface and that in the volume, as the size of an object decreases. When two surfaces are brought together, attractive (or repulsive) forces act between them, and a non-zero force is often required to separate two solid bodies placed in intimate contact [1 -7], a phenomenon referred to as adhesion.

Adhesion manifest itself in different ways. On one hand it makes it possible for a Gecko to walk on the ceilings or run on a vertical wall[ 8,9$]$. On the other hand, adhesion can lead to the failure of micro or nano devices, e.g. micro-sized cantilever beams [10]. Thus, if it is too long or too thin, the free energy minimum state corresponds to the cantilever beam (partly) bound to the substrate, which leads to the failure of the device. However, if the surface roughness is increased, the non-bonded cantilever state may be stabilized due to the decrease of cantileversubstrate binding energy.

Even the weakest force of interest in condensed matter physics, namely the van der Waals force, is relative strong on a macroscopic scale. Thus for two bodies with perfectly flat surfaces in contact over $1 \mathrm{~cm}^{2}$, a force equivalent to the weight of a car would be necessary to separate the surfaces even if only the weak van der Waals interaction act between the surfaces. In practice this is not the case, and this is referred to as the adhesion paradox. Thus the fundamental problem is not why adhesion sometimes is observed, but rather why it usually does not manifest itself in everyday life.

The explanation for the adhesion paradox is that no surface of practical use is perfectly flat but have surface roughness on many different length scale. In this case the interfacial bond breaking will not occur uniformly over the contacting interface, but will start at some defect and spread by interfacial crack propagation. The high stress concentration at the tip of cracks result in a much smaller force necessary for the separation of the surfaces than would be expected in the ideal case of perfectly smooth surfaces and uniform bond breaking.

In a classical study, Fuller and Tabor [6] have studied the influence of surface roughness on adhesion. They used silicon rubber balls in contact with (Plexiglas) surfaces with different amount of surface roughness produced by sand blasting. They found that with increasing surface roughness, the pull-off force dropped rapidly to zero, and using elastically softer rubber resulted in stronger adhesion than for stiffer rubber. This experimental work, and other similar studies [7], have stimulated much work to understand the role of roughness on adhesion.

Many practical application of adhesion involves soft elastic solids, e.g., pressure sensitive adhesives usually consist of weakly crosslinked rubber, which may exhibit very complex processes during pull-off such as cavitation and stringing [11, 12]. Here we consider the influence of surface roughness on adhesion for the most simple case of a purely elastic solid in contact with a hard randomly rough surface. If this simplest case cannot be understood in detail there is no hope to understand much more complex systems involving complex rheological materials in contact with randomly rough surfaces.

In order for two elastic solids with rough surfaces to make adhesive contact it is necessary to deform the surfaces elastically, otherwise they would only make contact in three points and the adhesion would vanish, at least if the spatial extent of the adhesion forces is neglected. Deforming the surfaces to increase the contact area $A$ result in some interfacial bonding $-\Delta \gamma A$ (where $\Delta \gamma=\gamma_{1}+\gamma_{2}-\gamma_{12}$ is the change in the interfacial energy per unit area upon contact), but it cost elastic deformation energy $U_{\mathrm{el}}$ which will reduce the effective binding. That is, during the removal of the block from the substrate the elastic compression energy stored at the interface is given back and helps to break the adhesive bonds in the area of real contact. Most macroscopic solids does 
not adhere with any measurable force, which imply that the total interfacial energy $-\Delta \gamma A+U_{\text {el }}$ vanish, or nearly vanish in most cases. However, not all the stored elastic energy $U_{\text {el }}$ may be used to break adhesive bonds during pull-off but some fraction of it may be radiated as elastic waves (phonons) into the solids. This would result in an increase in the effective interfacial binding energy, and would result in adhesion hysteresis.

In this letter we study the variation of the effective interfacial binding energy with the surface roughness amplitude, for elastic solids with randomly rough surfaces. We assume perfect (linear) elasticity and compare the results of MD-simulations for nominally flat and curved surfaces with a recently developed contact mechanics theory 13 16. The theoretical and simulation results agree rather well, and most of the differences observed can be attributed to finite size effects and to the longrange nature of the interaction between the atoms in the block and the substrate in the MD model, as compared to the analytical theory which is for an infinite system with interfacial contact interaction. For curved surfaces (JKR-type of problem) the effective interfacial energy exhibit a weak hysteresis which may be due to the influence of local irreversible detachment processes. This study represents the first test of the theory prediction for the effective adhesion energy for 3D systems [17].

We review the contact mechanics theory of Persson briefly. It can be used to calculate the stress distribution at the interface, the area of real contact and the average interfacial separation between the solid walls [13, 14]. In this theory, the interface is studied at different magnifications $\zeta=L / \lambda$ where $L$ is the linear size of the system and $\lambda$ the resolution. The wavevectors are defined as $q=2 \pi / \lambda$ and $q_{L}=2 \pi / L$ so that $\zeta=q / q_{L}$.

Consider an elastic block with a flat surface in adhesive contact with a hard substrate with a randomly rough surface. Let $\sigma(\mathbf{x}, \zeta)$ denote the (fluctuating) stress at the interface between the solids when the system is studied at the magnification $\zeta$. The distribution of interfacial stress

$$
P(\sigma, \zeta)=\langle\delta(\sigma-\sigma(\mathbf{x}, \zeta))\rangle
$$

In this definition we do not include the $\delta(\sigma)$-contribution from the non-contact area.

For perfect (or complete) contact it is easy to show that $P(\sigma, \zeta)$ satisfies 14 ]

$$
\frac{\partial P}{\partial \zeta}=f(\zeta) \frac{\partial^{2} P}{\partial \sigma^{2}}
$$

where

$$
f(\zeta)=\frac{\pi}{4} E^{* 2} q_{L} q^{3} C(q) .
$$

Here $E^{*}=E /\left(1-\nu^{2}\right)$ is the effective elastic modulus. The surface roughness power spectrum

$$
C(q)=\frac{1}{(2 \pi)^{2}} \int d^{2} x\langle h(\mathbf{x}) h(\mathbf{0})\rangle e^{-i \mathbf{q} \cdot \mathbf{x}}
$$

where $z=h(\mathbf{x})$ is the surface height at the point $\mathbf{x}=$ $(x, y)$ and where $\langle.$.$\rangle stands for ensemble average. The$ basic idea is now to assume that (2) holds locally also for incomplete contact.

To solve (2) one needs boundary conditions. If we assume that, when studying the system at the lowest magnification $\zeta=1$ (where no surface roughness can be observed, i.e., the surfaces appear perfectly smooth), the stress at the interface is constant and equal to $p=$ $F_{\mathrm{N}} / A_{0}$, where $F_{\mathrm{N}}$ is the load and $A_{0}$ the nominal contact area, then $P(\sigma, 1)=\delta(\sigma-p)$. In addition to this "initial condition" we need two boundary conditions along the $\sigma$-axis. Since there can be no infinitely large stress at the interface we require $P(\sigma, \zeta) \rightarrow 0$ as $\sigma \rightarrow \infty$. For adhesive contact, which interests us here, tensile stress occurs at the interface close to the boundary lines of the contact regions. In this case we have the boundary condition $P\left(-\sigma_{\mathrm{a}}, \zeta\right)=0$, where $\sigma_{\mathrm{a}}>0$ is the largest tensile stress possible. The detachment stress $\sigma_{\mathrm{a}}(\zeta)$ depends on the magnification and can be related to the effective interfacial energy (per unit area) $\gamma_{\text {eff }}(\zeta)$ using the theory of cracks [16]

$$
\sigma_{\mathrm{a}}(\zeta) \approx\left(\frac{\gamma_{\mathrm{eff}}(\zeta) E q}{1-\nu^{2}}\right)^{1 / 2}
$$

where

$$
\gamma_{\mathrm{eff}}(\zeta) A^{*}(\zeta)=\Delta \gamma A^{*}\left(\zeta_{1}\right)-U_{\mathrm{el}}(\zeta)
$$

where $A^{*}(\zeta)$ denotes the total contact area at the magnification $\zeta$, which is larger than the projected contact area $A(\zeta) . U_{\mathrm{el}}(\zeta)$ is the elastic energy stored at the interface due to the elastic deformation of the solids on length scale shorter than $\lambda=L / \zeta$, necessary in order to bring the solids into adhesive contact (see below).

From (1) it follows that the area of apparent contact (projected on the $x y$-plane) at the magnification $\zeta, A(\zeta)$, normalized by the nominal contact area $A_{0}$, can be obtained from

$$
\frac{A(\zeta)}{A_{0}}=\int_{-\sigma_{\mathrm{a}}(\zeta)}^{\infty} d \sigma P(\sigma, \zeta)
$$

We denote $A(\zeta) / A_{0}=P_{p}(q)$, where the index $p$ indicates that $A(\zeta) / A_{0}$ depends on the applied squeezing pressure $p$. The area of (apparent) contact at the highest magnification $\zeta=\zeta_{1}$ gives the real contact area. For the elastic energy $U_{\text {el }}$ we use 18$]$

$$
U_{\mathrm{el}} \approx A_{0} E^{*} \frac{\pi}{2} \int_{q_{L}}^{q_{1}} d q q^{2} W(q, p) C(q),
$$

where $q_{L}$ and $q_{1}$ are the smallest and the largest surface roughness wave vectors, and [18]

$$
W(q, p)=P_{p}(q)\left[\beta+(1-\beta) P_{p}^{2}(q)\right],
$$


where $\beta=0.45$. The equations given above are solved as described in Ref. [15].

Let us provide some details about the numerical simulations. The molecular dynamics system has lateral dimension $L_{x}=N_{x} a$ and $L_{y}=N_{y} a$, where $a$ is the lattice spacing of the block. In order to accurately study contact mechanics between elastic solids, it is necessary to consider that the thickness of the block is (at least) of the same order of the lateral size of the longest wavelength roughness on the substrate. We have developed a multiscale MD approach to study contact mechanics [19]. Periodic boundary condition has been used in $x y$ plane. For the block $N_{x}=N_{y}=512$, while the lattice space of the substrate $b=a$. The mass of the block atoms is 197 a.m.u. and the $a=2.6 \AA$. The elastic modulus and Poisson ratio of the block are $E=77.2 \mathrm{GPa}$ and $\nu=0.42$. For self-affine fractal surfaces, the power spectrum has power-law behavior $C(q) \sim q^{-2(H+1)}$, where the Hurst exponent $H$ is related to the fractal dimension $D_{\mathrm{f}}$ of the surface via $H=3-D_{\mathrm{f}}$. For real surfaces this relation holds only for a finite wave vector region $q_{0}<q<q_{1}$. Note that in many cases, there is a rolloff wave vector $q_{0}$ below which $C(q)$ is approximately constant. Here $q_{L}=2 \pi / L, q_{0}=3 q_{L}, q_{1}=512 q_{L}$. The physical meaning is that by choosing $q_{0}=3 q_{L}$ one can obtain a self-average equivalent to an average over 9 independent samples. In MD simulations, the substrate is rigid and fractal with fractal dimension $D_{\mathrm{f}}=2.2$ and root-mean-square roughness is varied from $h_{\mathrm{rms}}=0.1 \mathrm{~nm}$ to $2.5 \mathrm{~nm}$. We also studied adhesion where the substrate was curved into a nominally spherical cup with the radius of curvature $R=4410 \AA$. The calculations are carried out at temperature $T=0 \mathrm{~K}$.

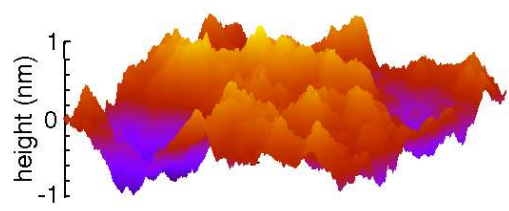

FIG. 1: The surface topography of a randomly rough surface with the root-mean-square roughness $0.3 \mathrm{~nm}$ and the Hurst exponent $H=0.8$ (i.e., fractal dimension $D_{\mathrm{f}}=2.2$ ).

The atoms at the interface between block and substrate interact with the potential

$$
V(r)=4 \epsilon\left[\left(\frac{r_{0}}{r}\right)^{12}-\left(\frac{r_{0}}{r}\right)^{6}\right]
$$

where $r$ is the distance between the pair of atoms. The parameter $\epsilon$ is the binding energy between two atoms at separation $r=2^{1 / 6} r_{0}$. In the calculations presented below we have used the $r_{0}=3.28 \AA$ and $\epsilon=100.0 \mathrm{meV}$. In order to calculate the interfacial binding energy, first we move the (upper surface) block at a low constant velocity towards substrate until the total force on the bot-

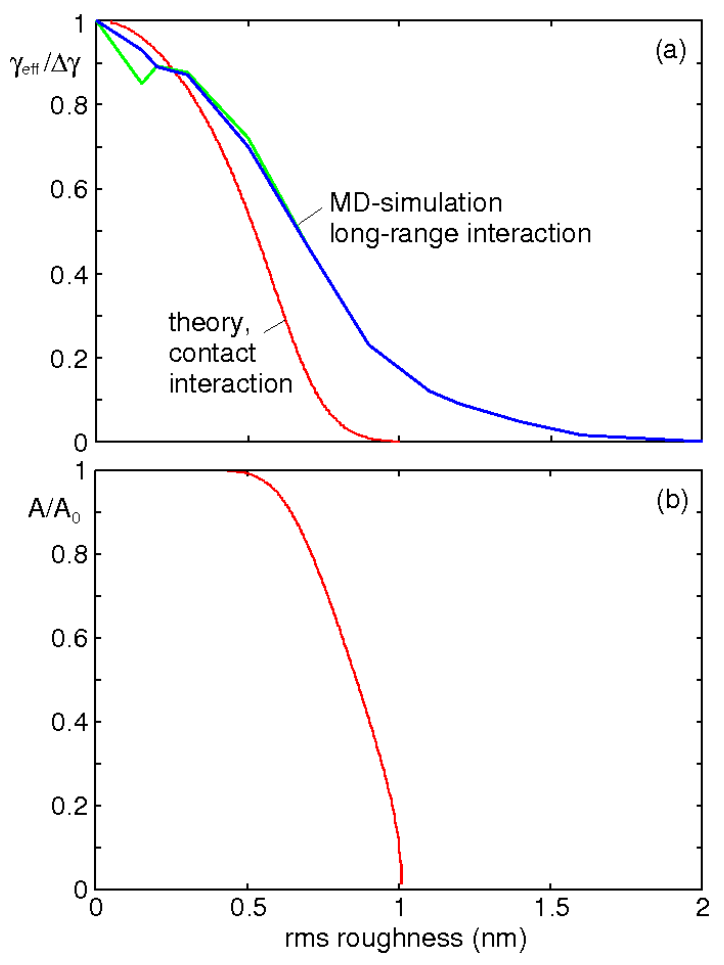

FIG. 2: (a) The interfacial binding energy $\gamma_{\text {eff }}$ (in units of the interfacial binding energy $\Delta \gamma=\gamma_{1}+\gamma_{2}-\gamma_{12}$ for flat surfaces) as a function of the root-mean-square roughness. The blue and green curves are the result of the MD-simulations obtained during contact formation (approach) and contact breaking (pull-off). The red curve is the theory prediction (using elastic continuum mechanics) for an infinite system with interfacial contact interaction. (b) The area of real contact $A$ as a function of the root-mean-square roughness calculated using the contact mechanics theory. The substrate surfaces are self-affine fractal (randomly rough) with the Hurst exponent $H=0.8$.

tom layer atoms of the block vanishes. At this point the work done by the substrate on the bottom layer of the block is defined as $\gamma_{\text {eff }} A_{0}$. In a similar way we calculate $\gamma_{\text {eff }}$ during the separation of the block from its equilibrium separation to infinite separation (pull-off). We find, as expected, that to within the accuracy of our calculations, $\gamma_{\text {eff }}$ obtained on approach and during separation are identical. For a flat substrate $\Delta \gamma$ turns out to be $\approx 100 \mathrm{meV} / \AA^{2}$.

In Fig. 2(a) we show the interfacial binding energy $\gamma_{\text {eff }}$, in units of the interfacial binding energy $\Delta \gamma=$ $\gamma_{1}+\gamma_{2}-\gamma_{12}$ for flat surfaces, as a function of the rootmean-square roughness. The blue and green curves are the result of the MD-simulations obtained during contact formation (approach) and contact breaking (pulloff). The red curve is the theory prediction (using elastic continuum mechanics) for an infinite system with wallwall contact interaction. The general shape of the curves are the same, but in the MD calculation the adhesion extend to larger surface roughness which we attribute to 
the small system size and the long range wall-wall interaction used in the MD model. That is, in the MD model there is an attractive force even between the non-contact surfaces which is absent in the continuum mechanics theory. This long-range attractive interaction is particular important for small systems and for surfaces with small amplitude roughness.

We note that the question of interaction energy at a distance is related to the difference between JKR [5] and DMT theories[20]. However, the situation is more complex in our case because here we have surface roughness which may generate "large" surface areas where the solid walls are closely spaced. In the JKR and DMT theories (involving smooth curved surfaces) the surfaces are only closely spaced close to the rim of the (circular) contact area. In our continuum mechanics theory we could in principle take (approximately) into account the interaction between the non-contact surface area using the (calculated) distribution of interfacial separations $P(u)[21]$.

In Fig. 2(b) we show the calculated area of real contact $A$, at the point where the external load vanish, as a function of the root-mean-square roughness calculated using the contact mechanics theory. Note that, as expected, $A$ vanish for the same roughness amplitude where $\gamma_{\text {eff }}=0$.

In an earlier paper 7] it has been shown that the pulloff force between silicon rubber balls and surfaces with different types of (non-fractal) roughness could be explained by a very simple theory where $\gamma_{\text {eff }}$ was calculated assuming that complete contact $\left(A=A_{0}\right)$ occur between the rubber and the substrate in the nominal (or apparent) contact area. Only for large roughness, where the pull-off force was $\sim 20 \%$ or less of the pull-off for the flat substrate, did this approach underestimate the pulloff force. This is consistent with the theory presented above. Thus in Fig. 3 we show the interfacial binding energy $\gamma_{\text {eff }}$ as a function of the root-mean-square roughness using the full theory (red line, from Fig. 2), and the result obtained assuming full contact for all rms roughness values (green line). Only for $\gamma_{\text {eff }} / \Delta \gamma<0.2$ does the full-contact theory underestimate the interfacial binding energy, which is consistent with the experimental data presented in Ref. [7].

Most solid objects have some macroscopic curvature which must be taken into account when determining the force necessary to separate two solids in adhesive contact. The theoretically most well defined situation is the contact between a ball and a flat surface. In this case, if at least one of the solids is elastically soft enough, the so called JKR theory can be applied, which predict the pull-off force to be 5 ]

$$
F=\frac{3 \pi}{2} \gamma_{\text {eff }} R
$$

In this equation $\gamma_{\text {eff }}$ is the effective interfacial energy (usually denoted as the work of adhesion) which includes the influence of the surface roughness on the interfa-

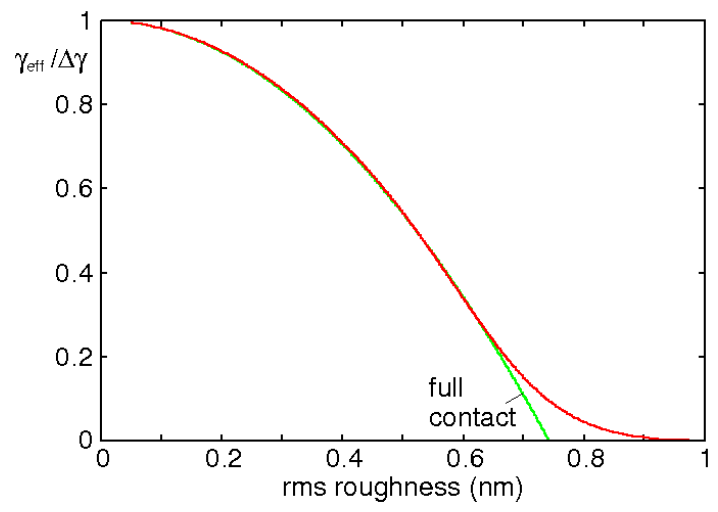

FIG. 3: The interfacial binding energy $\gamma_{\text {eff }}$ (in units of the interfacial binding energy $\Delta \gamma=\gamma_{1}+\gamma_{2}-\gamma_{12}$ for flat surfaces) as a function of the root-mean-square roughness. The red line is the full theory (from Fig. 2) while the green line is the result for the interfacial energy assuming full contact $\left(A=A_{0}\right)$ for all $\mathrm{rms}$ roughness values. (a)

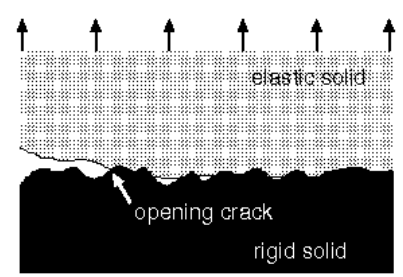

(b)

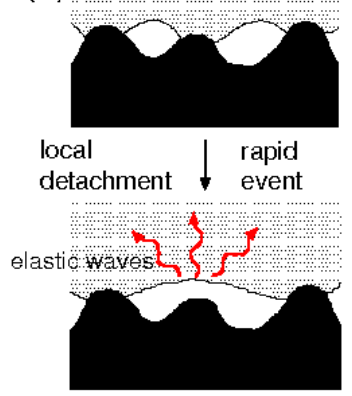

FIG. 4: (a) An opening of crack during pull-off. (b) A local detachment event in front of the crack tip. Asperity contact region detach rapidly with elastic energy radiating inside the block rather than being used to break other asperity contact regions (schematic).

cial binding energy. During pull-off the interfacial bondbreaking will not occur everywhere simultaneously, but a (circular) interfacial opening crack will propagate at the interface, see Fig. 4(a). The energy to propagate an interfacial crack may be larger than the energy necessary just to break the interfacial bonds. Thus, the work of adhesion which enter in (6) may be larger than the effective interfacial energy given by (3). For example, for rubber materials, unless the crack moves extremely slowly, there may be a large energy dissipation in the rubber close to the crack tip caused by the viscoelasticity of the rubber, which may enhance the force necessary for pull-off by several order of magnitudes. This effect can be taken into

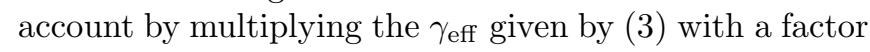
$1+f(v, T)$, where $f(v, T)$ is caused by viscoelastic energy dissipation in front of the crack tip, which depends on the crack tip velocity $v$ and the temperature $T[22,23]$. And 
even for perfectly elastic solids the work of adhesion may be larger than given by (3) since the separation of the surfaces at the crack tip may involve rapid events caused by local elastic instabilities close to the crack tip. Thus, for example, if a low asperity makes contact with the rubber the rubber may suddenly detach when the tensile stress from the approaching crack becomes high enough, see Fig. 4(b). If the elastic energy stored in the vicinity of the contact area before detachment is radiated as elastic waves into the block, rather than used to break other asperity contact regions, then this process will enhance the work of adhesion $\gamma_{\text {eff }}$ and the pull-off force. We will denote processes of the type illustrated in Fig. 4(b) as local irreversible detachment processes. This mechanism has recently been suggested to enhance the work of adhesion, but the study in Ref. [24] is for a periodic surface profile (sinus corrugation) and it is not cleat to what extent the same effect contribute for randomly rough surface.

We have studied the influence of rapid local detachment processes (Fig. 匹(b)) on the pull-off force for the same system as above but for a curved substrate surface. Thus we have curved the substrate surface so that it becomes a spherical cup with the radius of curvature $R=4410 \AA$, but the surface roughness is the same as for the nominally flat surfaces used above. We have measured the force on the block during approach and separation of the two solids, and used the JKR theory to determine the effective interfacial energy $\gamma_{\text {eff }}$. This approach includes the influence of local irreversible detachment processes (Fig. 4(b)). As above, the two solids were first approached to the point where the total force on the block vanished, and then separated. We found that if the upper surface of the block moves with the velocity $v=5 \mathrm{~m} / \mathrm{s}$ or less, the force on the block as a function of the (average) block-substrate separation was independent of $v$ and the results presented below was obtained with $v=5 \mathrm{~m} / \mathrm{s}$. We also tested that the damping which acted on the atoms (to remove elastic waves emitted from the interface) was so small that it had no influence on the pull-off force. For the curved surface without surface roughness we obtained from the maximum adhesion force using the JKR formula, for both the approach and the separation, the same interfacial energy $\gamma_{\mathrm{eff}}=\Delta \gamma \approx 100 \mathrm{meV} / \AA^{2}$ as determined above for the flat surfaces from the work done by the substrate on the bottom layer of the block.

In Fig. 5 we show the interfacial binding energy $\gamma_{\text {eff }}$ (in units of the interfacial binding energy $\Delta \gamma=\gamma_{1}+\gamma_{2}-\gamma_{12}$ for flat surfaces) as a function of the root-mean-square roughness. The red, blue and green lines are from Fig. 2. The pink and black curves (denoted "in" and "out") are the effective interfacial energy during approach and during separation, respectively, for the nominally curved (spherical cup) substrate (the curves was obtained from the maximum adhesion force using the JKR theory). Note that local irreversible detachment processes (in

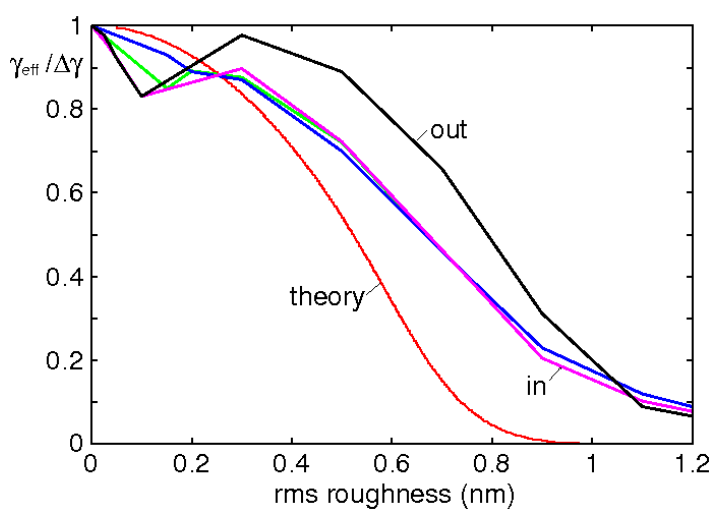

FIG. 5: The interfacial binding energy $\gamma_{\text {eff }}$ (in units of the interfacial binding energy $\Delta \gamma=\gamma_{1}+\gamma_{2}-\gamma_{12}$ for flat surfaces) as a function of the root-mean-square roughness. The red, blue and green lines are from Fig. 2 The pink and black curves (denoted "in" and "out") are the effective interfacial energy during approach and during separation, respectively, for the nominally curved (spherical cup) substrate (the curves was obtained from the maximum adhesion force using the JKR theory). The substrate surfaces are self-affine fractal (randomly rough) with the Hurst exponent $H=0.8$.

front of the crack tip) give a relative small increase in the pull-off force for randomly rough surfaces, and have a negligible influence on the force on the block during contact formation (approach).

The rapid decrease in $\gamma_{\text {eff }}$ observed in the MD simulations for small surface roughness ( $\mathrm{rms}<0.1 \mathrm{~nm}$ ) may reflect atomistic binding effects. That is, the minimum energy state for the flat surfaces correspond to the block atoms occupying hollow sites on the substrate. For the rough surface other binding configurations may form, e.g., domain-wall structures which would tend to increase the elastic energy stored at the interface and hence reduce $\gamma_{\text {eff }}$. Such atomistic effects are, of course, not included in the elastic continuum model.

Fig. 6] shows snapshot pictures of the contact region during pull-off for contact between the curved substrate with the rms roughness $3 \AA[(a)$ and (b)] and $9 \AA$ [(c) and (d)]. Fig. 6(a) and (c) are for the point where the total force on the block vanish and (b) and (d) for $0.14 \mathrm{~ns}$ later where the upper surface of the block has moved $7 \AA$ upwards. Note that the contact region for the rms roughness $3 \AA$ is compact, i.e., $A / A_{0}=1$ where $A_{0}$ is the nominal contact area, while for the rms roughness $9 \AA$ the contact is incomplete. This is consistent with the theory prediction [see Fig. 2(b)] which shows complete contact for the rms roughness $3 \AA$ but only partial contact for the rms roughness $9 \AA$.

Finally, we note that according to the continuum mechanics theory (see, e.g., [15]), adhesive contact mechanics depends on the dimensionless parameter $\theta=$ $E^{*} h_{\mathrm{rms}}^{2} q_{0} / \Delta \gamma$ and we could have plotted $\gamma_{\text {eff }} / \Delta \gamma$ as a function of $\theta$ instead of $h_{\mathrm{rms}}$ to emphasize the 

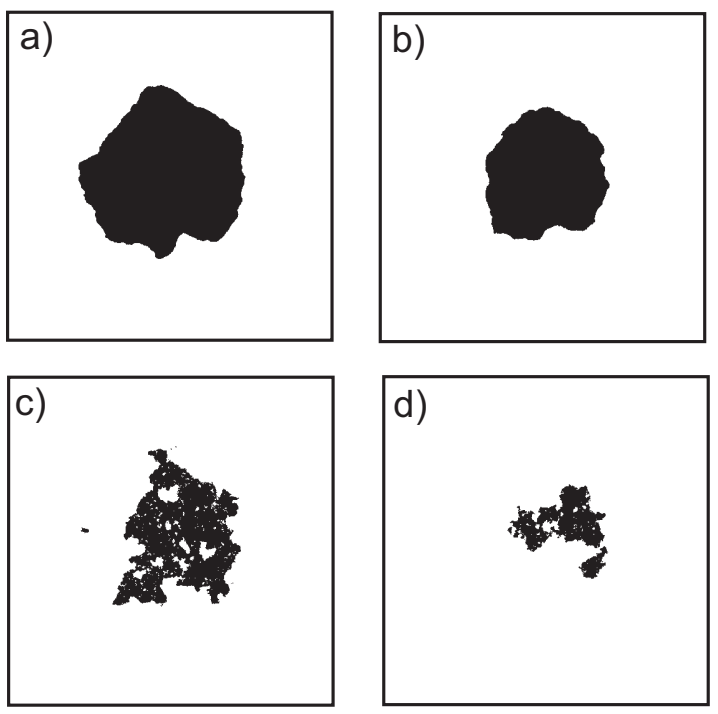

FIG. 6: Snap shot pictures of the contact region during pulloff for contact between the curved substrate with the rms roughness $3 \AA[(\mathrm{a})$ and (b)] and $9 \AA[(\mathrm{c})$ and (d)] and block surface. Pictures (a) and (c) are for the point where the total force on the block vanish and (b) and (d) for $0.14 \mathrm{~ns}$ later where the upper surface of the block has moved $7 \AA$ upwards.

more general or universal nature of our results. Of course, the result also depends on the detailed form of the surface roughness power spectra used. The MD model is atomistic and depends on an additional dimension less number, namely the Tabor number 25] $\mu=$ $R^{1 / 3} \Delta \gamma^{2 / 3} E^{-2 / 3} r_{0}^{-1}$, where $r_{0}$ is an atomic bond distance. Estimation of the Tabor number for our case shows that for the curved surface $\mu \approx 5$ so we are basically in the JKR-regime and therefore the pull-off force is given to a good approximation by Eq. (6) rather than the result of Derjaguin (valid for rigid solids) $F=2 \pi R \gamma_{\mathrm{eff}}$, or some intermediate value (e.g., DMT-theory) [26]. This is also confirmed by the fact that for smooth surfaces the $\Delta \gamma$ deduced for the flat substrate surface is the same as the $\Delta \gamma$ deduced from the JKR-formula for curved substrate surface (if the Derjaguin theory would instead be valid, the $\Delta \gamma$ deduced using the JKR theory should be a factor of $4 / 3$ times larger than deduced for the flat surface).

To summarize, we have presented a molecular dynamics (MD) study of the adhesive contact between elastic solids with randomly rough surfaces. We have calculated the interfacial binding energy and compared the results with the predictions of a recently developed contact mechanics model, which is based on continuum mechanics. There is good general agreement between the MD-results and the continuum mechanics theory, and the observed differences can be attributed to finite size effects and to the long range wall-wall interaction used in the MD simulation, in contrast to the infinite system size and contact interaction assumed in the theory. We have found that for randomly rough surfaces local irreversible detachment processes (in front of the crack tip) have a relative small influence on the pull-off force and a negligible influence on the force on the block during contact formation (approach).

[1] F.P. Bowden and D. Tabor, Friction and Lubrication of Solids (Wiley, New York, 1956).

[2] K.L. Johnson, Contact Mechanics, (Cambridge University Press, Cambridge, 1966).

[3] B.N.J. Persson, Sliding Friction: Physical Principles and Applications, 2nd edn. (Springer, Heidelberg, 2000).

[4] J.N. Israelachvili, Intermolecular and Surface Forces (Academic, London (1995)).

[5] K.L. Johnson, K. Kendall and A.D. Roberts, Proc. R. Soc. Lond. A. 324, 301 (1971)

[6] K.N.G. Fuller and D. Tabor, Proc. Roy. Soc. London A345, 327 (1975).

[7] A.G. Peressadko, N. Hosoda N and B.N.J. Persson, Phys. Rev. Lett. 95, 124301 (2005).

[8] H. Gao and H. Yao, PNAS101 7851 (2004)

[9] K. Autumn and N. Gravish, Phi. Tran. Roy. Sco. A.366, 1575 (2008)

[10] Y.P. Zhao, L.S. Wang and T.X. Yu, J. Ad. Sci. Tech.17, 519 (2003)

[11] A.N. Gent, P.B. Lindley, Proc. R. Soc. Lond. A 249, 195 (1958); A. N. Gent, D. A. Tompkins, J. Applied Phys. 40, 2520 (1969); Lakrout, Sergot and Creton, J. Adhesion 69, 307 (1999); Gay and Leibler, Phys. Rev. Lett. 82, 936 (1999); Poivet et al. Europhys. Lett. 62, 244 (2003); Eur. Phys. J. E15, 97 (2004); A. Zosel, Colloid and Polymer Sci. 263, 541 (1985); A. Zosel, J. Adhesion 30, 135 (1989) and Int. J. Adhesion \& Adhesives 18, 265 (1998); C. A. Dahlquist, Proc. Nottingham Conf. on Adhesion, 1966 Fundamental and Practice (MacLaren and Sons, Ltd. London); C. A. Dahlquist, in Treatise on Adhesion and Adhesives, R. L. Patrick (ed.), Dekker, New York (1969), 2.

[12] B.N.J. Persson, A. Kovalev, M. Wasem, E. Gnecco and S.N. Gorb, EPL 92, 46001 (2010).

[13] B.N.J. Persson, Phys. Rev. Lett. 99, 125502 (2007)

[14] B.N.J. Persson, J. Chem. Phys. 115, 3840 (2001).

[15] B.N.J. Persson, Eur. Phys. J E8, 385 (2002).

[16] B.N.J. Persson, Surface Science Reports 61, 201 (2006).

[17] G. Carbone, M. Scaraggi and U. Tartaglino, Eur. Phys. J E30, 65 (2009).

[18] C. Yang and B.N.J. Persson, J. Phys. Condens. Matter 20, 215214 (2008).

[19] C. Yang, U. Tartaglino and B.N.J. Persson, Eur. Phys. J. E19 , 47 (2006)

[20] D. Maugis J. Coll. Interf. Sci. 150(1) 243-269, 1992

[21] A. Almqquist, C. Campana, N. Prodanov and B.N.J. Persson, J.Mech. Phys. Solids 59, 2355 (2011).

[22] A.N. Gent and J. Schultz, J. Adhesion 3, 281 (1972).

[23] D. Maugis and M. Barquins, J. Phys. D 11, 1989 (1978).

[24] Q. Li and K-S Kim, Acta Mechanica Solida Sinica 22, 377 (2009).

[25] D. Tabor, J. Colloid Interface Sci. 58, 2 (1977).

[26] K.L. Johnson and J.A. Greenwood, J. Colloid Interface Sci. 192, 326 (1997). 